Provided for non-commercial research and education use. Not for reproduction, distribution or commercial use.

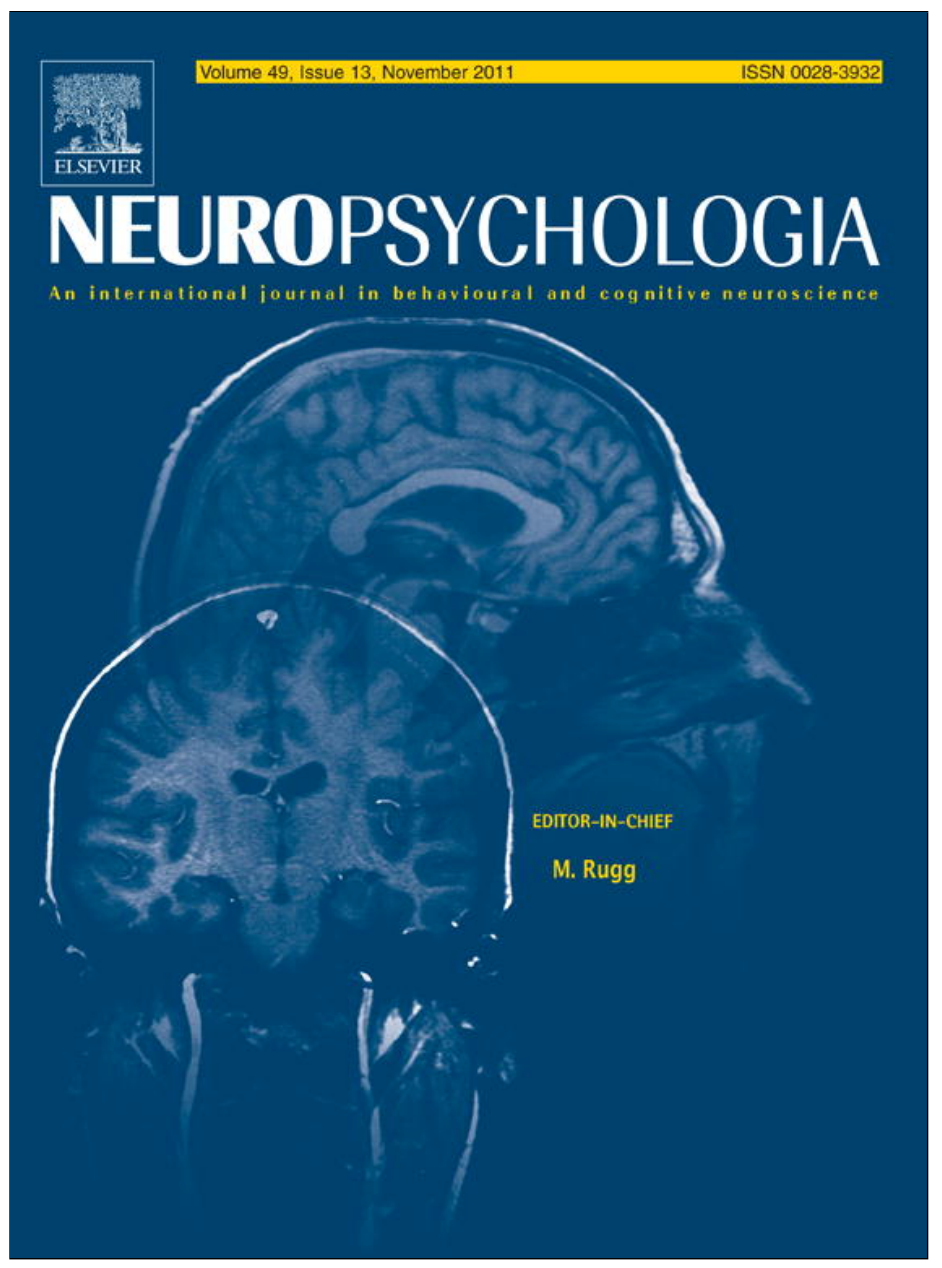

This article appeared in a journal published by Elsevier. The attached copy is furnished to the author for internal non-commercial research and education use, including for instruction at the authors institution and sharing with colleagues.

Other uses, including reproduction and distribution, or selling or licensing copies, or posting to personal, institutional or third party websites are prohibited.

In most cases authors are permitted to post their version of the article (e.g. in Word or Tex form) to their personal website or institutional repository. Authors requiring further information regarding Elsevier's archiving and manuscript policies are encouraged to visit:

http://www.elsevier.com/copyright 


\title{
Relearning in semantic dementia reflects contributions from both medial temporal lobe episodic and degraded neocortical semantic systems: Evidence in support of the complementary learning systems theory
}

\author{
Emily J. Mayberry, Karen Sage, Sheeba Ehsan, Matthew A. Lambon Ralph* \\ Neuroscience and Aphasia Research Unit (NARU), School of Psychological Sciences, University of Manchester, Manchester, UK
}

\section{A R T I C L E I N F O}

\section{Article history:}

Received 27 October 2010

Received in revised form 23 June 2011

Accepted 8 September 2011

Available online 16 September 2011

\section{Keywords:}

Semantic memory

Anterior temporal lobes

Relearning

Over-generalisation

Under-generalisation

\begin{abstract}
A B S T R A C T
When relearning words, patients with semantic dementia (SD) exhibit a characteristic rigidity, including a failure to generalise names to untrained exemplars of trained concepts. This has been attributed to an over-reliance on the medial temporal region which captures information in sparse, non-overlapping and therefore rigid representations. The current study extends previous investigations of SD relearning by re-examining the additional contribution made by the degraded cortical semantic system. The standard relearning protocol was modified by careful selection of foils to show that people with semantic dementia were sometimes able to extend their learning appropriately but that this correct generalisation was minimal (i.e. the patients under-generalised their learning). The revised assessment procedure highlighted the fact that, after relearning, the participants also incorrectly over-generalised the learned label to closely related concepts. It is unlikely that these behaviours would occur if the participants had only formed sparse hippocampal representations. These novel data build on the notion that people with semantic dementia engage both the degraded cortical semantic (neocortex) and the episodic (medial temporal) systems to learn. Because of neocortical damage to the anterior temporal lobes, relearning is disordered with a characteristic pattern of under- and over-generalisation.
\end{abstract}

(c) 2011 Elsevier Ltd. All rights reserved.

\section{Introduction}

An important challenge which the brain must overcome when learning about the world is to integrate new information and experiences into an existing body of knowledge without over-writing previously learned concepts (McClelland, McNaughton, \& O'Reilly, 1995). For example, it is important that, if someone sees "Fido", a three-legged dog, this experience does not change their understanding that dogs generally have four legs and equally, they need to recognise that this atypical exemplar remains a dog.

\subsection{The complementary learning systems theory}

This challenge (avoiding catastrophic interference while still learning from novel experiences: McClelland et al., 1995; Rogers \& McClelland, 2004) can be overcome by the brain making use of two learning systems: one which can learn about particular episodes

\footnotetext{
* Corresponding author at: Neuroscience and Aphasia Research Unit (NARU), Zochonis Building, School of Psychological Sciences, University of Manchester, Oxford Road, Manchester M13 9PL, UK. Tel.: +44 161275 2551; fax: +441612752873

E-mail address: matt.lambon-ralph@manchester.ac.uk (M.A. Lambon Ralph).
}

or items quickly and the other which is slower to learn but integrates these experiences effectively into a long-term database. The Complementary Learning Systems theory (McClelland et al., 1995) sets out a neural division of labour between the hippocampus (and adjacent medial temporal lobe structures) and the neocortex. These learning systems are highly interconnected but represent information in different ways - sparse representations which enable quick learning in the hippocampal system and integrated, generalisable representations which take more time to become embedded in the neocortical system. The two systems work in tandem to build up the distributed neocortical representations at the appropriate rate; each activation in the fast-learning system leads to a slight change in the weighting of information within the slower neocortical learning system. This division of labour allows for the optimal balance between maintaining the fidelity of the recently experienced item/episode and integration into a generalisable database while not over-writing previously learned information.

\subsection{Neuropsychological evidence for each of the two systems}

The hippocampal system is crucial for new declarative learning (see Squire, 1992 for a review; see McClelland et al., 1995) and is particularly involved in new episodic learning. Single unit recordings in rats suggest that hippocampal representations are relatively 
sparse, with a small percentage of neurons firing in a given situation and place cells within hippocampal neurons firing selectively to represent a specific pattern of cues (Barnes et al., 1990; Quirk, Muller, \& Kubie, 1990). The consequences of sparse hippocampal representations for learning are that situations which differ only slightly may share relatively little representational overlap (Marr, 1969; McNaughton \& Morris, 1987; Randall and James, 1994). This allows for specific pieces of information to be captured quickly and stored relatively independently from other memories but, as a consequence, does not allow for similarities between different items or experiences to be captured. Sparse learning is particularly useful for episodic memory (remembering particular events or experiences) because each life experience needs to be kept distinct from other events.

One of the most striking sources of evidence for the role of the sparse representational, fast-learning hippocampal system in the initial stages of declarative learning comes from studies of people who have bilateral hippocampal damage (Isaacs et al, 2003; Scoville \& Milner, 1957; Squire \& Knowlton, 1995; Vargha-Khadem et al., 1997). After bilateral hippocampal resection, people are unable to form new memories and lose recently acquired information. Most famously, patient HM had severe anterograde amnesia following a bilateral hippocampal resection but intact intellectual function and sparing of remote memories (Scoville \& Milner, 1957). HM could learn new motor skills (e.g. mirror tracing) but had no recollection of ever performing the tasks before. Each time he returned to the clinic, he did not recognise the staff or the tasks he had carried out on previous visits, even if the interval between visits was a matter of days. However, because his conceptual knowledge was intact, he could recognise, appropriately name and use items he had learnt about prior to his surgery (e.g. mirror, pen, clinic, chair, table, etc.).

In contrast to the medial temporal system, the neocortex is slower to learn but builds up distributed representations such that new information is integrated with all previously learned knowledge and can be appropriately generalised (McClelland et al., 1995). As well as representing specific information about each concept, semantic memory licenses a range of key capacities including appropriate generalisation of information across concepts and representation of shared structure (Lambon Ralph, Sage, Jones, \& Mayberry, 2010; Mayberry, Sage, \& Lambon Ralph, 2011). While a distributed set of cortical regions code different modality-specific sources of information, there is now convergent evidence that the anterior temporal regions play a key role in the formation of "coherent" concepts by coding across modality-invariant representations (Binney, Embleton, Jefferies, Parker, \& Lambon Ralph, 2010; Pobric, Jefferies, \& Lambon Ralph, 2010). This region is the focus of atrophy and hypometabolism in semantic dementia (Galton et al., 2001; Nestor, Fryer, \& Hodges, 2006) and these patients present with a selective semantic impairment. Accordingly, this patient group provides a key source of evidence not only about the breakdown of existing (consolidated) semantic knowledge but also about the role played by this system in new learning. Specifically, the patients provide a direct counterpoint to the medial temporal lobe dysfunction found in classical amnesia (see above) and thus provide insights about the neocortical component of the Complementary Learning Systems theory.

\subsection{Evidence for interconnectivity of the two systems}

The Complementary Learning Systems theory (McClelland et al., 1995) also suggests that there is a gradual shift in the division of labour over time with respect to the contribution of both memory systems to declarative memory. The hippocampal system is critical during the initial stages of declarative learning (Scoville \& Milner, 1957) and becomes less involved as time passes, as the information becomes better represented in other neocortical brain regions (Squire, 1992; Takashima et al., 2006, 2009). A recent illustration of this functional shift comes from a sleep study (Takashima et al., 2009) which showed that recalling recently learned information (in a recognition memory task using photographs of natural landscapes) primarily activated the hippocampus. After more time had passed and the participants had slept, the hippocampus was less active and neocortical regions were more active during recall of the information. Lesion studies of learning in non-human primates have also illustrated this phenomenon; for example, when monkeys learned object pair discrimination (Zola-Morgan \& Squire, 1990), those monkeys who were in the control group (no hippocampal lesion after learning) performed better on the recently learned object discrimination trials than on the earlier learned object discrimination trials, indicating that recent information was remembered more clearly than information learned earlier. However, the monkeys in the experimental group (hippocampal lesions after learning) showed the reverse pattern; they performed worse on recently learned trials than earlier learned trials. In addition, monkeys in the lesion group performed equally to non-lesioned monkeys on the earlier learned trials, indicating that this earlier learned information was no longer represented in the hippocampus and instead was reliant on other brain regions. These results indicate a selective role of the hippocampal system in representing recently acquired memories and a gradual shift in the neural basis of these representations to the neocortex for longer-term storage.

With regard to the interaction between the two systems, Kan, Alexander, and Verfaellie (2009) showed that amnesic patients with hippocampal damage and varying degrees of ATL damage were able to learn new episodic information (in spite of the hippocampal damage) but that the degree of ATL damage modulated their ability to learn new episodic information. People with less ATL damage (and thus more baseline knowledge about the study topic) were able to learn new episodic information better than people with more extensive ATL damage. This indicates that the cortical system provides top-down support for episodic learning in addition to the contribution made by the hippocampal system.

A key target for the present study was to investigate whether a related phenomenon might hold for patients with SD - that is to say, their relearning would be influenced by the partially degraded, neocortical semantic representations. This hypothesis arises from the repeated observations, across numerous receptive and expressive semantic tasks, that concepts are not lost wholesale in SD but rather the representations degrade in a very graded fashion (Hodges, Graham, \& Patterson, 1995; Rogers et al., 2004; Warrington, 1975). One might expect, therefore, that while the semantic-cortical contribution to new learning might decrease in semantic dementia (as demonstrated in previous relearning studies - see below), there would still be some contribution to learning/re-learning from cortical-based semantic representations. Accordingly, the changing quality of the semantic input to learning should reflect the nature of the degraded cortical concepts.

\subsection{Semantic generalisation: A crucial role of the anterior temporal lobes}

Past relearning studies have shown that people with semantic dementia can relearn the precise mappings between items and their corresponding names but that the learning is very rigid (e.g. Graham, Patterson, Pratt, \& Hodges, 1999, 2001; Heredia, Sage, Lambon Ralph, \& Berthier, 2009; Jokel, Rochon, \& Leonard, 2006; Snowden \& Neary, 2002). In all of these studies, the patients failed to generalise the trained label to untrained exemplars of the same concept (under-generalise), unless they were very visually similar to the trained targets (for example, after relearning the name for a pictured banana, patient CUB was able to extend the label to a 
visually similar depiction but not to sliced bananas or a bunch of the fruit: Heredia et al., 2009).

This rigid learning/under-generalisation has been attributed to an over-reliance on hippocampal learning (Graham et al., 1999; Snowden \& Neary, 2002). This follows directly from the complementary learning systems hypothesis because, as noted above, medial temporal lobe representations are sparse and keep newly formed memories insulated from each other. As a consequence, these representations do not license generalisation. In addition, however, several lines of evidence suggest that the remaining, degraded (neocortical) semantic knowledge may contribute to and generate the nature of relearning observed in SD. First, as described above, across a variety of verbal and nonverbal semantic tasks it has been demonstrated that the SD patients continue to activate the remnants of their conceptual knowledge automatically, implying that this degraded information has the potential to influence non-semantic processes including the fast-learning hippocampal system (Adlam et al., 2006; Hodges et al., 1995). Secondly, and in keeping with this assumption, studies which have explored the role of baseline knowledge in relearning have found that SD patients demonstrate better relearning of names for which they have greater remaining conceptual knowledge (Graham et al., 2001; Jokel et al., 2006; Snowden \& Neary, 2002). Finally, two recent explorations of SD have found that in semantic matching-to-sample assessments (which entail no learning or relearning but simply probe the nature of the patients' remaining semantic knowledge using carefully selected materials), the patients made two types of errors: (1) they over-generalised - incorrectly including items which are semantically similar to the target but are not exemplars of the target concept (e.g. include a Chihuahua when given the category label of 'cat') and (2) they simultaneously under-generalised - failing to include target exemplars which are atypical of the target concept (e.g. fail to select a hairless, Sphinx cat as being a cat: Lambon Ralph et al., 2010; Mayberry et al., 2011). In contrast, the patients were unlikely to make errors to more distantly related (distant semantic and unrelated) items. These studies have argued that the ATL contribution to semantic representation may be especially important for the computation of appropriate generalisations independent of superficial similarities (arguably a core function of semantic memory, see Lambon Ralph \& Patterson, 2008; Lambon Ralph et al., 2010; Mayberry et al., 2011; Pobric et al., 2010). In the context of the present study, these previous investigations are important in that they suggest that the patients' degraded semantic representations might act as a second source of under-generalisations in re-learning.

\subsection{Current study aims and objectives}

Past SD relearning studies have demonstrated undergeneralisation of relearning which suggests an over-reliance on the hippocampal learning system (Graham et al., 1999, 2001; Snowden \& Neary, 2002). However, there are at least two possible sources for this phenomenon which arise from the complementary learning systems hypothesis: (1) an over-reliance on the hippocampal system (caused by deterioration of the cortical semantic system); and (2) continued influence of the degraded ATL semantic hub. If the second explanation is correct, at least in part, then we would expect a combination of both under-generalisation errors after relearning (which could be promoted by both causes) and over-generalisation errors (caused by the degraded semantic system alone). The latter have not been reported in previous relearning studies but this may be due to the selection of pre- and post-relearning assessment materials. Specifically, most studies have probed naming performance of (a) the items used in the relearning phase; (b) untreated items matched for familiarity and other psycholinguistic properties; and sometimes (c) untreated additional exemplars of the trained items (e.g. a different depiction of the same concept). In this design, the untreated items (set B) rarely include semantically related concepts which are the most likely to elicit over-generalisation errors in verbal or picture-based matching-to-sample assessments (see Lambon Ralph et al., 2010; Mayberry et al., 2011). As a consequence, in this new relearning study, not only did we include two probes for the target to test for under-generalisation (the learned example and visually dissimilar untrained exemplar; e.g. an upright hoover vs. a Henry hoover) but also - as a test of over-generalisation - three untrained foil probes: (i) visually and semantically related items (e.g. a lawn mower); (ii) a distant semantic foil (e.g. a bin), and (iii) an unrelated picture (e.g. a yacht). This example is shown in Fig. 1. If the degraded semantic, neocortical system still engages during relearning in SD (as would be expected from the complementary learning systems hypothesis) then we would expect to see a combination of underand over-generalisations after relearning.

\section{Methods}

\subsection{Participants}

$\mathrm{NH}$ presented at the age of 65, with a 2-year history of word-finding difficulties and failure to recognise familiar people, including long-standing personal acquaintances. Initial clinical assessment confirmed significant anomia, surface dyslexia, person recognition deficits but good orientation for time and place, episodic memory and visuospatial skills. An initial CT scan noted greater right than left temporal lobe atrophy consistent with her clinical presentation, including features of progressive prosopagnosia. A provisional diagnosis of semantic dementia was made and Alzheimer's disease was rejected. Our initial neuropsychological screen confirmed these clinical features, consistent with semantic dementia. $\mathrm{NH}$ was well orientated in time and place, was able to recall accurate details from personal events and recent news stories, and was able to navigate around her local environment without error. She organised her own diary, weekly routines and needed no assistance with her medications or their administration. In addition to her prosopagnosia, $\mathrm{NH}$ presented with surface dyslexia, profound anomia and clear multi-modal comprehension deficits. In contrast her repetition, syntax and other aspects of her language were preserved. These features of selective semantic impairment are highly characteristic of semantic dementia. In addition, $\mathrm{NH}$ presented with mild disinhibition and variable impulsiveness on formal testing - suggesting features of a more frontotemporal pattern. $\mathrm{NH}$ was reviewed clinically on a six-monthly basis for the next two years. Her semantic impairment continued to evolve but remained selective - with preservation in other aspects of cognitive and everyday functioning. She organised not only her own medication but also the administration of her husband's. She also began to read her junk mail obsessively and developed somatic complaints, for which she was separately treated. Our neuropsychological re-assessments confirmed NH's worsening, yet still selective, multimodal semantic impairment.

GE presented at the age of 53 with an 18 month history of progressive wordfinding difficulties and difficulties understanding words in conversations. He had left full-time employment 12 months previously due to these difficulties but continued to work part-time. He was well oriented in time and place, was able to drive and navigate by himself, and could recall details from recent events. Our initial neuropsychological screening confirmed these features. GE presented with good episodic memory, visuospatial skills, but had significant anomia, multimodal semantic impairment and surface dyslexia. Over the next three years, GE's semantic impairment and word-finding difficulties progressed but these problems remained selective with minimal changes on any of the other language and cognitive measures.

Both NH's and GE's scan highlighted bilateral anterior temporal lobe atrophy including atrophy to the temporal poles and the inferior surface of the temporal lobes. NH's pattern of atrophy was bilateral with slightly more pronounced right ATL damage, while GE's scan showed more left than right sided ATL atrophy. Neither NH nor GE had significant generalised atrophy and the scans showed relative preservation of the medial temporal lobes for both participants. Brain scans are shown for NH (CT) and GE (MRI) in Fig. 2.

At the time of this study, both patients completed a range of background assessments to ascertain the degree of global semantic impairment and the preservation of other cognitive skills. The results summarised in Table 1 show that they were both impaired on semantic tasks (e.g. naming, word-to-picture matching, semantic association judgements) while able to perform within the normal range on various tasks that tap perceptual and visuospatial processes, and other non-semantic tests. It is also worth noting that neither patient made visually related naming errors, in keeping with a semantic rather than perceptual deficit. At the time of the current study, NH had a milder semantic impairment than GE. 


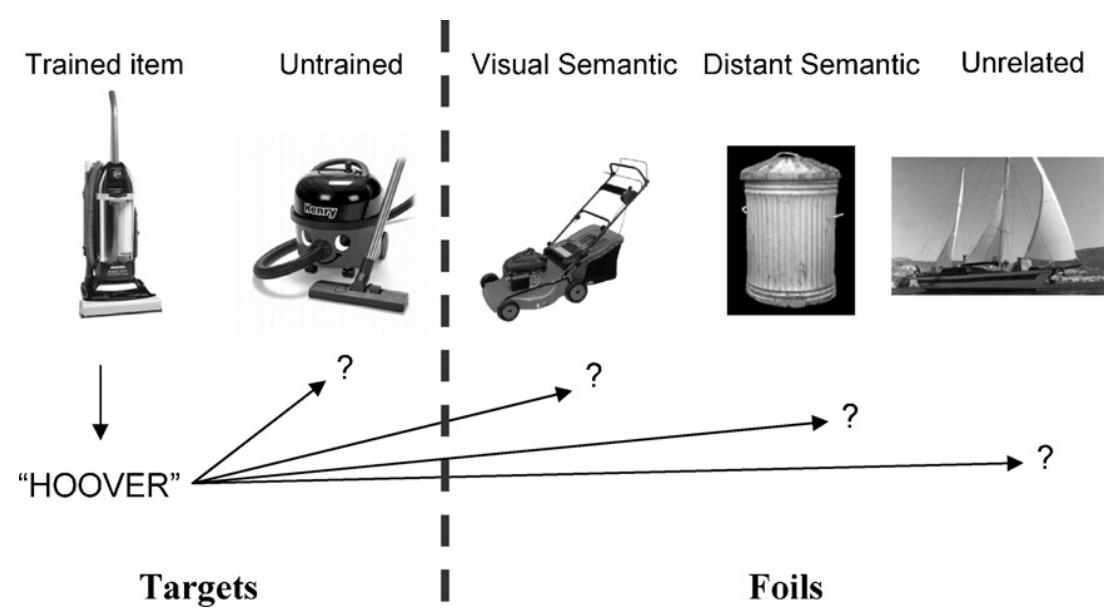

Fig. 1. The relearning paradigm. Footnote: an example of the items used in this relearning study. The standard upright vacuum cleaner was the trained exemplar (typically named as a "hoover" in the UK) and the four other concepts were used to probe naming performance before and after the training. These included an alternative exemplar of the same concept (to detect under-generalisation errors) and three different concepts which varied in conceptual similarity to the trained item. Following previous semantic matching-to-sample assessments (Lambon Ralph et al., 2010; Mayberry et al., 2011) we expected that over-generalisation errors would be most likely for the close visual-semantic concepts.

\subsection{Stimuli}

Thirty items to be trained were selected from a range of categories which included animals (including fish and birds), household items, clothing, and food items. A list of the 30 exemplars, their visually unrelated targets and the three types of associated foils are provided in Appendix 1. Thus, in total there were 150 picture stimuli.

\subsection{Learning method and testing schedule}

At the baseline assessment, $\mathrm{NH}$ and GE attempted to name the 150 items presented in random order in colour photo format. Their responses were coded as correct and the errors classified (omission, semantic including coordinate or superordinate, or other). After baseline testing, both $\mathrm{NH}$ and GE received a booklet with the 30 items to be trained. Each item was displayed first as a picture (e.g. the whole galia melon) and then on the next page, the same picture was presented with the written label below. For each item, the participant was encouraged to look at the first page (picture only) and to attempt to name it. If they were unable to name the picture, then they were instructed to turn the page and read the answer aloud. The initial session was carried out in the home with one of the researchers to demonstrate the procedure. After that, the training regime was carried out daily for three weeks by phone with the same researcher in the exact same manner. Responses were recorded for every session.
Immediately after the three weeks of daily practice, another home visit was made and all 150 items were tested again, in the same order as they had been at baseline (not the same as the studied order). The study booklets were then set aside for one month during which no practice took place. A further retest of all 150 of the items was carried out at the end of that month to determine how much learning had been maintained without continued practice.

\section{Results}

\subsection{Accuracy for trained items}

At baseline naming, $\mathrm{NH}$ named just over a third of the 150 items correctly $(55 / 150,36.7 \%)$, in line with her mild to moderate semantic impairment. NH's errors for the set as a whole $(n=95)$ were divided between semantic errors (60/95, 63.2\%) - including superordinate $(21 / 60)$, coordinate $(31 / 60)$, and some incomplete semantic descriptions (8/60) - and omission errors (23/95, 24.2\%). Other errors were rare $(12 / 95,12.6 \%)$. For the set of 30 trained items, NH's errors $(n=19)$ were categorised in the same way. $\mathrm{NH}$ made a large proportion of semantic errors $(9 / 19,47.4 \%)$ including

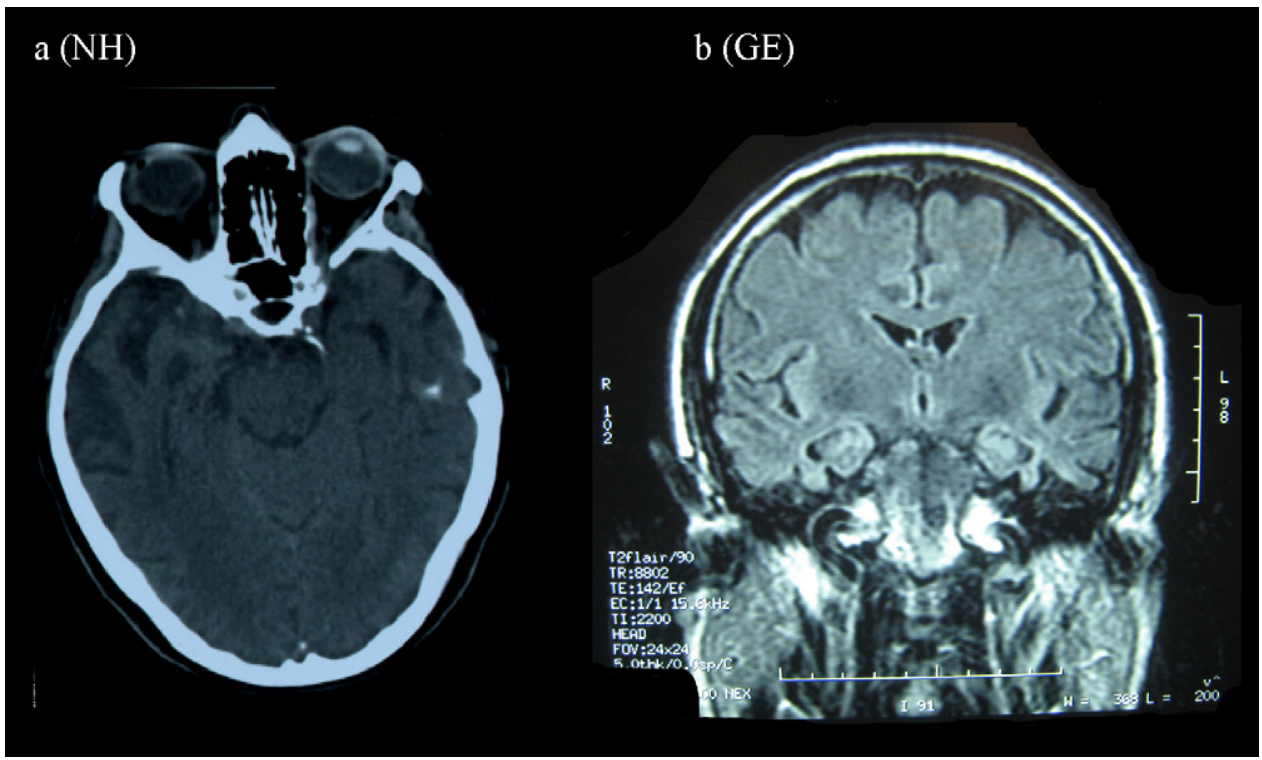

Fig. 2. Brain scans for $\mathrm{NH}$ (axial CT) and GE (coronal MRI). 
Table 1

Background semantic and cognitive testing for two participants with SD.

\begin{tabular}{|c|c|c|c|c|}
\hline & & & $\mathrm{NH}$ & GE \\
\hline Age & & & 65 & 52 \\
\hline Sex & & & $\mathrm{F}$ & M \\
\hline Scan & & & Bilateral ATL atrophy, $R>L$ & Bilateral ATL atrophy, $L>R$ \\
\hline Semantic tests & Max score & Normal cut off/range & $\mathrm{NH}$ & GE \\
\hline 64 naming & 64 & 59 & $24^{\mathrm{a}}$ & $14^{\mathrm{a}}$ \\
\hline 64 WPM & 64 & 63 & $37^{\mathrm{a}}$ & $34^{\mathrm{a}}$ \\
\hline PPT pictures & 52 & 49 & NT & $35^{\mathrm{a}}$ \\
\hline PPT words & 52 & 49 & NT & $33^{\mathrm{a}}$ \\
\hline CCT pictures & 64 & 53 & $41^{\mathrm{a}}$ & $25^{\mathrm{a}}$ \\
\hline CCT words & 64 & 56 & NT & $22^{\mathrm{a}}$ \\
\hline Category fluency ( 8 categories) & $\mathrm{n} / \mathrm{a}$ & 89 & $14^{\mathrm{a}}$ & $22^{\mathrm{a}}$ \\
\hline \multicolumn{5}{|l|}{ Perceptual and visuospatial tasks } \\
\hline VOSP dot counting & 10 & 8 & 10 & 10 \\
\hline VOSP cube analysis & 10 & 6 & 9 & 10 \\
\hline VOSP position discrimination & 20 & 18 & $16^{\mathrm{a}}$ & 20 \\
\hline VOSP number location & 10 & 7 & 10 & 10 \\
\hline Rey figure copy & 36 & $\%$ & NT & $>99$ th \%。 \\
\hline \multicolumn{5}{|l|}{ Other cognitive tests } \\
\hline Ravens coloured progressive matrices & 36 & $\%$ & 25 th \%。 & $>95$ th \%。 \\
\hline Digit span (forward) & $\mathrm{n} / \mathrm{a}$ & 5 & $4^{\mathrm{a}}$ & 7 \\
\hline Letter fluency & $\mathrm{n} / \mathrm{a}$ & 22 & 34 & $19^{a}$ \\
\hline TEA elevator counting (without distraction) & 7 & 6 & 7 & 7 \\
\hline TEA elevator counting (with distraction) & 10 & 3 & 9 & 10 \\
\hline
\end{tabular}

F, female; M, male; R, right hemisphere; L, left hemisphere; ATL, anterior temporal lobes; NT, not tested; 64 naming, Cambridge 64 item naming test (Bozeat, Lambon Ralph, Patterson, Garrard, \& Hodges, 2000); 64 WPM, Cambridge 64 item word-to-picture matching (Bozeat et al., 2000); PPT, Pyramids and Palm Trees test of semantic association (Howard \& Patterson, 1992); CCT, Cambridge 64 item Camel and Cactus Test of semantic association (Bozeat et al., 2000); category fluency, how many concepts from a particular category (eight tested) could be produced in one minute; Ravens coloured progressive matrices (Raven, 1962); complex figure of Rey copy (Osterrieth, 1995); digit span (Wechsler, 1987); letter fluency, how many words beginning with a particular letter (F, A, S tested) could be produced in one minute; VOSP, Visual Object and Space Perception battery (Warrington \& James, 1991); TEA, Test of Everyday Attention elevator counting subtest (Robertson, Ward, Ridgeway, \& Nimmo-Smith, 1994).

a Person's score is below the normal cut-off on that particular test.

superordinate (4/9), coordinate (4/9), and some incomplete semantic descriptions (1/9). She also made a large number of omission errors $(8 / 19,42.1 \%)$ and a very small number of other errors $(2 / 19$, $10.5 \%)$.

At baseline, GE named fewer items (23/150, 15.3\%) than NH, in line with his moderate to severe semantic impairment. Similarly to $\mathrm{NH}$, most of GE's errors $(n=127)$ were semantic $(89 / 127$, $70.1 \%$ ) including superordinate (35/89), coordinate (20/89), and some incomplete semantic descriptions (34/89). He also made some omission errors $(21 / 127,16.5 \%)$ and a small number of other errors $(17 / 127,13.4 \%)$. For the set of 30 trained items, GE's errors $(n=25)$ were categorised in the same way. GE made a large proportion of semantic errors $(17 / 25,68.0 \%)$ including superordinate (11/17), coordinate (2/17), and some incomplete semantic descriptions (4/17). He also made some omission errors (5/25, 20.0\%) and a small number of other errors $(3 / 25,8.0 \%)$ on the set of trained items.

Fig. 3 shows the significant improvements both NH (Fig. 3a) and GE (Fig. 3b) made immediately after training (McNemar, onetailed, $p<0.001$ baseline to re-test). At the follow-up one month after learning had ceased, both participants maintained the benefit of relearning, with no decline in performance ( $\mathrm{NH} \mathrm{30/30} \mathrm{at} \mathrm{both}$ time points and GE 29/30 at both time points).

\subsection{Evidence of appropriate generalisation and under-generalisation}

$\mathrm{NH}$ did not correctly generalise any of the additional, newly relearnt names to the visually dissimilar untrained targets at retest (McNemar two-tailed, $p=0.625$ ) or follow-up (McNemar two-tailed $p=0.250$ ). In comparison to NH's complete undergeneralisation of her learning, GE did generalise at least some of the trained labels to the untrained targets (McNemar two-tailed $p=0.021)$ and this generalisation was maintained at the follow-up (13/30 re-test and follow-up). However, in spite of demonstrating some appropriate generalisation, neither NH nor GE showed full appropriate generalisation of the learned labels.

\subsection{Evidence of over-generalisation}

Over-generalisation was assessed by examining how often each participant incorrectly used the trained label when shown the 90 foils at re-test (see Fig. 4). This was compared against baseline because a small rate of apparent over-generalisation errors would be expected by chance (SD patients make coordinate semantic errors in spontaneous naming and occasionally these might happen to match one of the closely related foils). For $\mathrm{NH}$, there was a significant change in her incorrect use of trained labels (over-generalisations) for the foils as a whole (McNemar onetailed, $p=0.035$ ) and this was carried by a borderline increase in over-generalisations for the visual-semantic foils (McNemar one-tailed, $p=0.063$ ). There was no change in the rate of overgeneralisations for the distant semantic or the unrelated foils (both McNemar one-tailed $p=1.00$ ). GE was also significantly more likely to incorrectly over-generalise the trained labels after relearning for the foils as a whole (McNemar one-tailed $p<0.001$ ) and for the visual-semantic foils (McNemar one-tailed $p<0.001$ ). There was no significant change in over-generalisations for the distant semantic foils (McNemar one-tailed $p=0.313$ ) and unrelated foils (McNemar one-tailed $p=0.063$ ).

\section{Discussion}

This study explored the outcome of name relearning in two people with semantic dementia. In particular, this study focussed not only on the under-generalisation of this kind of relearning but also 
a

(patient NH)

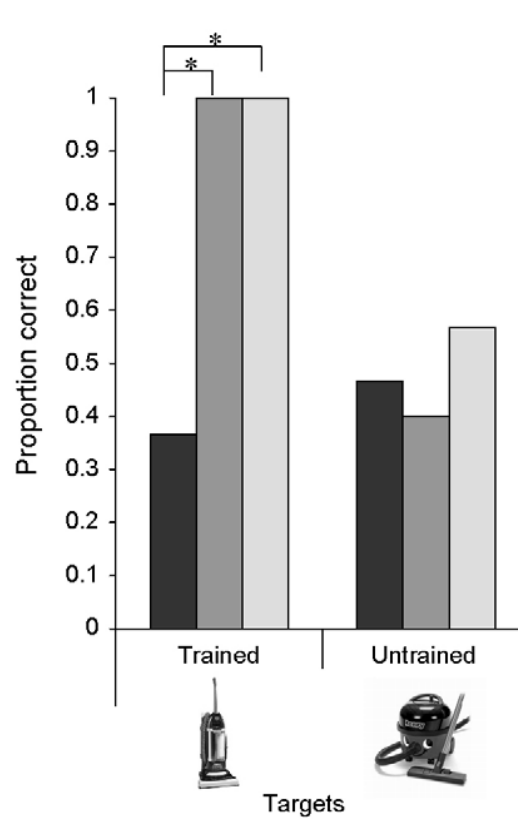

b (patient GE)

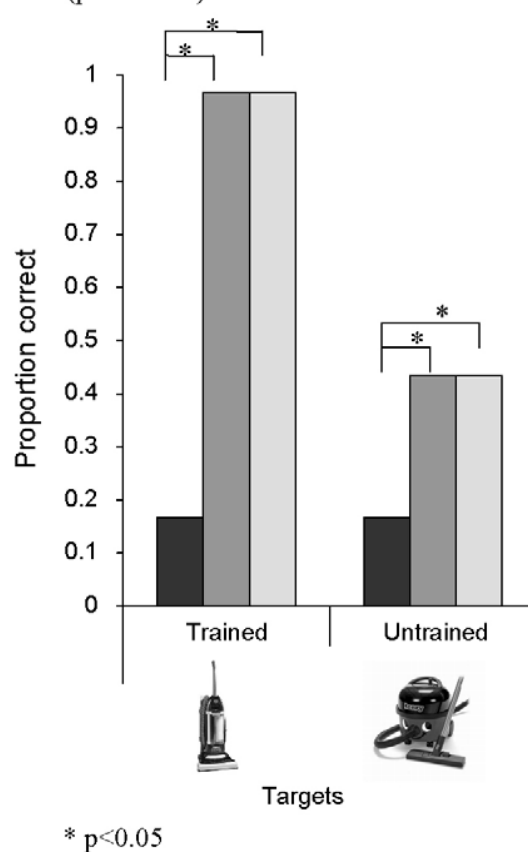

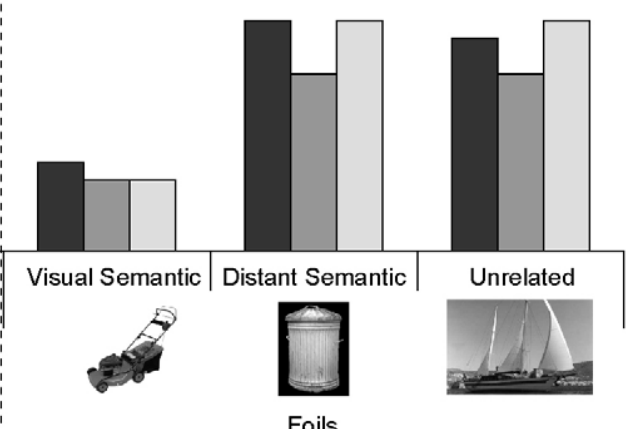

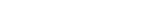

Fig. 3. Naming accuracy at baseline, post-relearning and follow-up testing.

on if and when the relearning also leads to over-generalisations. This would be expected from the complementary learning systems hypothesis but has never been reported in any previous SD relearning investigations (Beir et al., 2009; Bozeat, Patterson, \& Hodges, 2004; Dewar, Patterson, Wilson, \& Graham, 2009; Frattali, 2004; Funnell, 1995; Graham et al., 1999, 2001; Henry, Beeson, \& Rapcsak, 2008; Heredia et al., 2009; Jokel, Rochon, \& Anderson, 2010; Jokel et al., 2006; Robinson, Druks, Hodges, \& Garrard, 2009; Snowden \& Neary, 2002). Just like previous studies, the two patients' naming of the target, trained items improved significantly after three weeks of daily practice and they maintained these improvements one month later. Although GE demonstrated a significant improvement in naming the untrained, alternative target exemplars, this appropriate generalisation was limited and was non-existent in
NH's performance. An additional, novel observation was that both patients tended to over-generalise the relearnt names to concepts that were semantically and superficially similar. In contrast, these errors were not found for more distant, semantically related or unrelated items (which correspond to the type of non-trained, control items included in most previous relearning studies).

Under-generalisation has been noted in many other SD relearning studies (Graham et al., 1999, 2001; Snowden \& Neary, 2002) and was attributed to a sole reliance on the sparse representational hippocampal system. While this may well account for some of the patients' under-generalisations, it is important to note that, as predicted, there was evidence of at least some continued contribution from the patients' degraded (neocortical) semantic system; both participants incorrectly over-generalised the retrained names to 


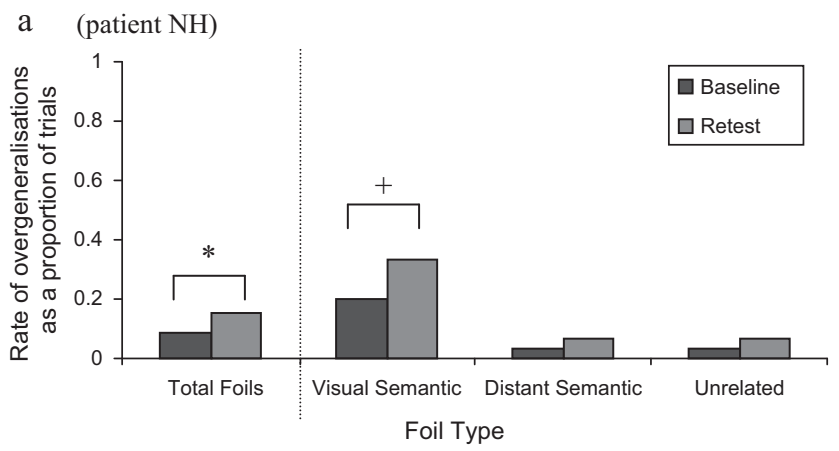

b (patient GE)

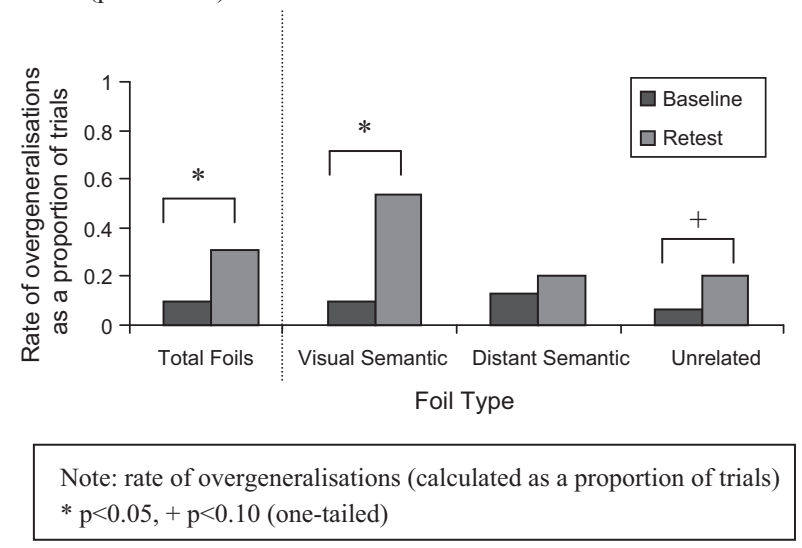

Fig. 4. Proportion of over-generalisation errors at baseline and at retest.

the closely related semantic foils (mirroring the coordinate semantic errors observed in spontaneous picture naming) and in GE's case, there was some limited correct generalisation to visually dissimilar target exemplars. As noted in Section 1, hippocampal representations are assumed to be sparse in nature and this has the benefit of licensing rapid consolidation of new information, without confusing it with any previous experiences. As a consequence, this system offers no basis on which the new information can be generalised (unless the precise form and characteristics of the stimuli and event re-occur). The relative contributions of the hippocampal and neocortical components of the CLS following relearning cannot be completely differentiated based on the results of this study (as under-generalisations could be explained by learning via either system); however, the finding of over-generalisations shows that the degraded semantic system is still involved.

An alternative explanation for over-generalisation errors following relearning is that they are based purely on visual similarity between the trained targets and the closely related semantic foils. However, there are several reasons why this explanation is unlikely. First, people with SD do not have perceptual impairments. In spite of their semantic deficits, they perform within control ranges on tasks which require them to discriminate between items based on perceptual characteristics (Graham, Simons, Pratt, Patterson, \& Hodges, 2000; Green \& Patterson, 2009; Piwnica-Worms, Omar, Hailstone, \& Warren, 2010). Indeed, both NH and GE performed very well on the background tests of visual perceptual abilities (see Table 1). Secondly, further evidence that the over-generalisation errors do not occur as a result of a perceptual problem comes from a recent study of semantic processing in SD (Mayberry et al., 2011). This study showed that people with SD both overgeneralise and under-generalise semantic information even when the testing format does not provide any perceptual information about the concepts. Specifically, the same pattern of over- and under-generalisation errors was shown when the participants were tested either with pictures or only verbal stimuli (written words, which were read aloud by the experimenter). This and another recent study (Lambon Ralph et al., 2010) demonstrated that people with SD increasingly rely on the superficial rather than deep conceptual similarity structure. This was true even when the information was probed with written words. Given that, unlike pictures, words and concepts have an entirely arbitrary relationship, there are no useful clues in the surface form of the word about its meaning and thus the observed over- and under-generalisation errors must have a semantic rather than visual locus.

The pattern of results found in the current study (particularly the novel observation of over-generalisation of the target names) is similar to a phenomenon illustrated by a computational model of the development of conceptual knowledge in children (Rogers \& McClelland, 2004). As such, this previous research provides a formal (semantic) explanation for over-generalisations not only in normal development but also, potentially, in SD relearning. In this model, concepts were formed through the gradual distillation of distributed semantic attributes, integrated across different contexts. As a result, conceptual representations were formed gradually and, like children, concepts which were not well differentiated early in the learning process were prone to over-generalisation. Specifically, Rogers and McClelland (2004) trained the computational model on a set of concepts and then, at various points during its 'development', they tested generalisation by training the model to associate a new property with one of the (semi-formed) concepts (e.g. the maple has a 'queem'). When fully developed, the model was able to generalise the new property appropriately to the other trees but not to other non-tree items. Earlier during development when the concepts were only semi-formed, however, the new attribute was incorrectly applied (over-generalised) to a large set of items across a variety of categories. It seems very likely that a similar explanation would hold for the over-generalisations found in SD relearning. Specifically, relearning the name of an item would involve re-strengthening the link between this verbal attribute and the underlying degraded semantic representation of the target concept. Just like semi-formed concepts, there would then be the inherent danger that this feature would be linked not only to the target item but also the most closely related semantic representations. As such, this approach would provide an explanation for the differences found between GE and $\mathrm{NH}$ in terms of the severity of their semantic impairment. The fact that GE demonstrated greater rates of over-generalisation fits with the fact that his semantic system was more impaired than $\mathrm{NH}$ and, as a consequence, there was a greater danger of confusing closely related concepts. We note here that an additional bonus of this explanation is that it brings together the phenomena of over-generalisation in relearning but also in the patients' impaired semantic performance (which involves no relearning) through a single explanation. The same, degraded semantic representations would not only produce over-generalisation in relearning but also the semantic coordinate naming errors and other types of over-generalisation errors found in various previous studies of comprehension and expressive semantic abilities (Lambon Ralph et al., 2010; Mayberry et al., 2011).

By way of conclusion, we finish by noting that these results have at least two clear clinical implications for the management of this patient group. Future relearning therapies for semantic dementia must consider and assess generalisation errors after relearning, as under- and over-generalisations will only be observed if materials are specifically designed to detect these error types. Secondly, future studies might try to develop relearning paradigms which will not only encourage people with SD to extend the trained information appropriately but also to constrain it based on semantic information rather than superficial similarities. 


\section{Acknowledgements}

We would like to thank GE and NH who took part in this study and their carers for their time and effort. We would also like to thank Dr. Andrew Larner for referring the patients to us. EJM was supported by an Overseas Research Student (ORS) Award and the University of Manchester. This project was supported by an MRC programme grant to MALR (G0501632).

\section{Appendix A. Supplementary data}

Supplementary data associated with this article can be found, in the online version, at doi:10.1016/j.neuropsychologia.2011.09.010.

\section{References}

Adlam, A. L. R., Patterson, K., Rogers, T. T., Nestor, P. J., Salmond, C. H., AcostaCabronero, J., et al. (2006). Semantic dementia and fluent primary progressive aphasia: two sides of the same coin? Brain, 129, 3066-3080.

Barnes, C. A., McNaughton, B. L., Mizumori, S. J. Y., Leonard, B. W. \& Lin, L.-H. (1990) Comparison of spatial and temporal characteristics of neuronal activity in sequentia stages of hippocampal processing. Progress in brain research Elsevier., pp. 287-300 (chap. 21)

Bier, N., Macoir, J., Gagnon, L., Van der Linden, M., Louveaux, S. \& Desrosiers, J. (2009) Known, lost, and recovered: Efficacy of formal-semantic therapy and spaced retrieval method in a case of semantic dementia. Aphasiology, 23(2), 210-235.

Binney, R. J., Embleton, K., Jefferies, E., Parker, G. J. M. \& Lambon Ralph, M. A. (2010) The inferolateral aspects of the anterior temporal lobe are crucial in semantic memory: Evidence from a novel direct comparison of distortion-corrected fMRI rTMS and semantic dementia. Cerebral Cortex.

Bozeat, S., Lambon Ralph, M. A., Patterson, K., Garrard, P. \& Hodges, J. R. (2000) Non-verbal semantic impairment in semantic dementia. Neuropsychologia, 38 1207-1215.

Bozeat S., Patterson, K. \& Hodges, J. (2004). Relearning object use in semantic dementia. Neuropsychological Rehabilitation: An International Journal, 14(3), 351-363.

Dewar, B.-K., Patterson, K., Wilson, B. A. \& Graham, K. S. (2009). Re-acquisition of person knowledge in semantic memory disorders. Neuropsychological Rehabilitation, 19(3), 383-421.

Frattali, C. (2004). An errorless learning approach to treating dysnomia in frontotemporal dementia. Journal of Medical Speech-Language Pathology, 12(3), XI-XXIV.

Funnell, E. (1995). A case of forgotten knowledge. In R. Campbell, M. Conway (Eds.) Broken memories (pp. 225-236).

Galton, C. J., Patterson, K., Graham, K. S., Lambon Ralph, M. A., Williams, G., Antoun, N., et al. (2001). Differing patterns of temporal atrophy in Alzheimer's disease and semantic dementia. Neurology, 57, 216-225.

Graham, K. S., Patterson, K., Pratt, K. H. \& Hodges, J. R. (1999). Relearning and subsequent forgetting of semantic category exemplars in a case of semantic dementia. Neuropsychology, 13(3), 359-380.

Graham, K. S., Patterson, K., Pratt, K. H. \& Hodges, J. R. (2001). Can repeated exposure to forgotten vocabulary help alleviate word-finding difficulties in semantic dementia? An illustrative case study. Neuropsychological Rehabilitation, 11(3-4), 429-454.

Graham, K. S., Simons, J. S., Pratt, K. H., Patterson, K. \& Hodges, J. R. (2000). Insights from semantic dementia on the relationship between episodic and semantic memory. Neuropsychologia, 38, 313-324.

Green, H. A. C. \& Patterson, K. (2009). Jigsaws-a preserved ability in semantic dementia. Neuropsychologia, 47, 569-576.

Henry, M. L., Beeson, P. M. \& Rapcsak, S. Z. (2008). Treatment for lexical retrieval in progressive aphasia. Aphasiology, 22(7-8), 826-838.

Heredia, C. G., Sage, K., Lambon Ralph, M. A. \& Berthier, M. (2009). Relearning and retention of verbal labels in a case of semantic dementia. Aphasiology, 23, 192-209.

Hodges, J. R., Graham, N. \& Patterson, K. (1995). Charting the progression of semantic dementia: Implications for the organisation of semantic memory. Memory, 3, 463-495.

Howard, D. \& Patterson, K. (1992). The Pyramids and Palm Trees Test: A test of semantic access from words and pictures. Bury St Edmunds: Thames Valley Test Company.

Isaacs, E. B., Vargha-Khadem, F., Watkins, K. E., Lucas, A., Mishkin, M. \& Gadian, D. G. (2003). Developmental amnesia and its relationship to degree of hippocampal atrophy. Proceedings of the National Academy of Sciences of the United States of America, 100(22), 13060-13063.

Jokel, R., Rochon, E. \& Anderson, N. D. (2010). Errorless learning of computergenerated words in a patient with semantic dementia. Neuropsychological Rehabilitation, 20(1), 16-41.
Jokel, R., Rochon, E. \& Leonard, C. (2006). Treating anomia in semantic dementia: Improvement, maintenance, or both? Neuropsychological Rehabilitation, 16 241-256.

Kan, I. P., Alexander, M. P. \& Verfaellie, M. (2009). Contribution of prior semantic knowledge to new episodic learning in amnesia. Journal of Cognitive Neuroscience, 21(5), 938-944.

Lambon Ralph, M. A. \& Patterson, K. (2008). Generalization and differentiation in semantic memory: Insights from semantic dementia. In A. Kingstone, \& M. Miller (Eds.), The year in cognitive neuroscience. NY: Annals of the NY Academy of Sciences.

Lambon Ralph, M. A., Sage, K., Jones, R. W. \& Mayberry, E. J. (2010). Coherent concepts are computed in the anterior temporal lobes. Proceedings of the National Academy of Sciences, 107(6), 2717-2722.

Marr, D. (1969). A theory of cerebellar cortex. The Journal of Physiology, 202(2), 437-470.

Mayberry, E. J., Sage, K. \& Lambon Ralph, M. A. (2011). At the edge of semantic space: The breakdown of coherent concepts following anterior temporal lobe atrophy is constrained by typicality and severity but not modality. Journal of Cognitive Neuroscience, 23, 2240-2251. doi:10.1162/jocn.2010.21582

McClelland, J. L., McNaughton, B. L. \& O'Reilly, R. C. (1995). Why there are complementary learning-systems in the hippocampus and neocortex: Insights from the successes and failures of connectionist models of learning and memory. Psychological Review, 102(3), 419-457.

McNaughton, B. L. \& Morris, R. G. M. (1987). Hippocampal synaptic enhancement and information storage within a distributed memory system. Trends in Neurosciences, $10(10)$, 408-415.

Nestor, P. J., Fryer, T. D. \& Hodges, J. R. (2006). Declarative memory impairments in Alzheimer's disease and semantic dementia. Neuroimage, 30(3), 1010-1020.

Osterrieth, P. (1995). Complex figure of Rey (3rd ed.). Oxford: OUP.

Piwnica-Worms, K. E., Omar, R., Hailstone, J. C. \& Warren, J. D. (2010). Flavour processing in semantic dementia. Cortex, 46(6), 761-768.

Pobric, G., Jefferies, E. \& Lambon Ralph, M. A. (2010). Category-specific versus category-general semantic impairment induced by transcranial magnetic stimulation. Current Biology, 20, 964-968.

Quirk, G. J., Muller, R. U. \& Kubie, J. L. (1990). The firing of hippocampal place cells in the dark depends on the rat's recent experience. Journal of Neuroscience, 10(6), 2008-2017.

Randall, C. O. R. \& James, L. M. (1994). Hippocampal conjunctive encoding, storage, and recall: Avoiding a trade-off. Hippocampus, 4(6), 661-682.

Raven, J. C. (1962). Coloured progressive matrices: Sets A, AB, B. London: HK Lewis.

Robertson, I., Ward, T., Ridgeway, V. \& Nimmo-Smith, I. (1994). The test of everyday attention. Bury St Edmunds, UK: Thames Valley Test Company.

Robinson, S., Druks, J., Hodges, J. \& Garrard, P. (2009). The treatment of object naming, definition, and object use in semantic dementia: The effectiveness of errorless learning. Aphasiology, 23(6), 749-775.

Rogers, T. T. \& McClelland, J. L. (2004). Semantic cognition: A parallel distributed processing approach. Cambridge, MA: The MIT Press.

Rogers, T. T., Lambon Ralph, M. A., Garrard, P., Bozeat, S., McClelland, J. L., Hodges, J. R. et al. (2004). The structure and deterioration of semantic memory: A neuropsychological and computational investigation. Psychological Review, 111, 205-235.

Scoville, W. B. \& Milner, B. (1957). Loss of recent memory after bilateral hippocampal lesions. Journal of Neurology, Neurosurgery and Psychiatry, 20(1), 11-21.

Snowden, J. S. \& Neary, D. (2002). Relearning of verbal labels in semantic dementia. Neuropsychologia, 40(10), 1715-1728.

Squire, L. R. (1992). Memory and the hippocampus: A synthesis from findings with rats, monkeys, and humans. Psychological Review, 99(2), 195-231.

Squire, L. R. \& Knowlton, B. J. (1995). Learning about categories in the absence of memory. Proceedings of the National Academy of Sciences of the United States of America, 92(26), 12470-12474

Takashima, A., Petersson, K. M., Rutters, F., Tendolkar, I., Jensen, O., Zwarts, M. J., et al. (2006). Declarative memory consolidation in humans: A prospective functional magnetic resonance imaging study. Proceedings of the National Academy of Sciences of the United States of America, 103(3), 756-761.

Takashima, A., Nieuwenhuis, I. L. C., Jensen, O., Talamini, L. M., Rijpkema, M. \& Fernandez, G. (2009). Shift from hippocampal to neocortical centered retrieval network with consolidation. Journal of Neuroscience, 29(32), 10087-10093.

Vargha-Khadem, F., Gadian, D. G., Watkins, K. E., Connelly, A., Van Paesschen, W. \& Mishkin, M. (1997). Differential effects of early hippocampal pathology on episodic and semantic memory. Science, 277(5324), 376-380.

Warrington, E. K. (1975). The selective impairment of semantic memory. Quarterly Journal of Experimental Psychology, 27(4), 635-657.

Warrington, E. K. \& James, M. (1991). The visual object and space perception battery. Bury St Edmunds: Thames Valley Test Company.

Wechsler, D. (1987). Wechsler memory scale-Revised (WMS-R). New York: Psychological Corporation.

Zola-Morgan, S. M. \& Squire, L. R. (1990). The primate hippocampal formation: Evidence for a time-limited role in memory storage. Science, 250(4978), 288-290. 